\title{
Growth in infinite groups of infinite subsets
}

\author{
J. O. Button \\ Selwyn College \\ University of Cambridge \\ Cambridge CB3 9DQ \\ U.K. \\ jb128@dpmms.cam.ac.uk
}

\begin{abstract}
Given an infinite group $G$, we consider the finitely additive measure defined on finite unions of cosets of finite index subgroups. We show that this shares many properties with the size of subsets of a finite group, for instance we can obtain equivalent results on Ruzsa distance and product free sets. In particular if $G$ has infinitely many finite index subgroups then it has subsets $S$ of measure arbitrarily close to $1 / 2$ with square $S^{2}$ having measure less than 1.
\end{abstract}

\section{Introduction}

The theory of the growth of finite subsets $A, B$ in a group $G$ is well established: one considers the size of the products sets $A B, A^{2}, B^{2}$ and higher powers in terms of $|A|$ and $|B|$. One hopes to be able to say something about the structure of such sets if any or all of $|A B|,\left|A^{2}\right|,\left|A^{3}\right|, \ldots$ are comparatively small. The subject originated with abelian groups but recently results have been established for non abelian groups too, as discussed in [23] and this will be our focus here.

One often takes $G$ to be finite, although in this context we do not really stick to one group but allow $G$ to vary over an infinite family of groups (such as the non abelian finite simple groups) or even over all finite groups. There are also results for infinite groups, where we might consider one group $G$ only 
or again a whole class. We just mention two recent results in this regard: the first, which is Theorem 2 in [6], covers torsion free groups $G$. It states that there exists a polynomial $p(n)=32(n+3)^{6}$ such that if $A$ is a finite subset of any torsion free group $G$ which is not contained in a left coset of a cyclic subgroup and $|B|>p(n)$ then $|A B|>|A|+|B|+n$. The other result is that of Safin in [20] which states that there is $c>0$ such that $\left|A^{3}\right| \geq c\left|A^{2}\right|$ for $A$ any finite subset of the non abelian free group $F_{2}$ of rank 2 with $\langle A\rangle$ not cyclic.

However if our group $G$ is infinite then no matter how quickly the sequence $\left|A^{n}\right|$ grows, we have that the proportion of elements of $G$ in $A^{n}$ is zero. We would like to assign size or measure to infinite subsets $A, B$ of $G$ and their product sets, in such a way that the measure of $G$ is finite in order to see if similar results apply. Of course there are several ways to do this, such as if $G$ is a compact group equipped with a Haar measure on the Borel subsets of $G$. However in this paper our groups of interest will often be countable, so any countably additive measure defined on all subsets of $G$ which is invariant under left multiplication will either assign 0 to all $g$ in $G$, and hence to $G$, or infinite measure to $G$.

A successful way round this for some countable discrete groups is to observe that asking for countable additivity is expecting too much and so one requires a finitely additive left invariant measure $\mu$ with $\mu(G)=1$ defined on all subsets of $G$, leading us to the important class of amenable groups. But many countable groups are not amenable, including any group containing the free group $F_{2}$ (the only specific group mentioned so far).

Another way out of this, at least for infinite groups $G$ which are finitely generated, is to work in the profinite completion $\hat{G}$ which is a compact group with Haar measure, obtained from the finite quotients of $G$. Our approach in this paper has connections with this, in that it also looks at the finite quotients of a group, but is much simpler. We have already mentioned that countable additivity is a very strong property to expect in a measure. Moreover so is having it defined on all subsets of a group, as we see with the reals and Lebesgue measure. Thus we adopt the more relaxed approach of merely requiring our measure to be finitely additive on a class of subsets which are closed under finite unions, finite intersections, and complementation. However we require some sets to be measurable, and any sensible notion of size should assign $1 / i$ to a coset $g H$ of a subgroup $H$ that has index $i$ in $G$. Therefore we leave it at that and only take finite unions of cosets of finite index subgroups for our measurable sets, forming the basic measure as defined 
in Section 2. Some of our results also hold for any left invariant measure extending the basic measure, which we term an acceptable measure.

In Section 3 we look at the standard concepts for product sets in finite groups, especially in the non abelian case as covered in [23] and featuring such topics as Ruzsa distance. We show that they easily generalise to any infinite group $G$ equipped with the basic measure. A big plus here is that, just as with finite sets, if $A$ is a subset of $B$ with the basic measure of $A$ equal to that of $B$ then $A=B$. This allows us as in the finite case to identify measurable subsets $A$ satisfying certain conditions on their measure, for instance in Corollary 3.9 where $A^{n}$ is a proper subset of $G$ for all integers $n$. We also point out that even for finite groups we can have generating sets $S$ which are symmetrised but with no $n$ such that $S^{n}=G$.

In Section 4 we are interested in comparing the size $|A|$ of a measurable subset $A$ with $\left|A^{2}\right|$. There has been much work done on this for finite groups and we first review such results, especially the problem of trying to find subsets $A$ with $A^{2} \neq G$ such that $|A|$ is as large as possible. We obtain Corollary 4.8 which says that if $G$ is an infinite finitely generated linear group in characteristic zero then there is always $A \subseteq G$ with $|A| /|G|=1 / 2$ but such that $A^{2} \neq G$. This is achieved by showing in Theorem 4.4 that the same statement is true when $G$ is any finite group of even order. Another example of a concept in finite groups that applies here is that of a product free subset $S$ of a group $G$, which is one where $S$ and $S^{2}$ do not intersect, and a recent result of Gowers shows that for any $\epsilon>0$ there is a finite simple group $G_{\epsilon}$ such that $|S| /|G|<\epsilon$ for any product free subset $S$ of $G_{\epsilon}$. We show that this is still true with $G_{\epsilon}$ an infinite finitely generated residually finite group by applying a construction of Kassabov and Nikolov which uses profinite groups.

In the last Section, we explore the fact that for any group $G$ Ruzsa distance is a genuine metric on the set $\mathcal{S}(G)$ of the finite index subgroups of $G$. Proposition 5.2 tells us when the triangle inequality becomes an equality, allowing us to turn this set into a metric graph. Consequently we have a conjugation action by isometries of $G$ on this graph and we show that this action is faithful if and only if $G$ is residually finite with trivial centre. 


\section{Definitions and results}

Definition 2.1 Given a set $\Omega$, an algebra $\mathcal{A}$ of $\Omega$ is a collection of subsets of $\Omega$ with $\Omega \in \mathcal{A}$ such that $A, B \in \mathcal{A}$ implies that $A \cup B$ and $A \backslash B \in \mathcal{A}$.

This implies that $\emptyset \in \mathcal{A}$. Moreover if $A_{1}, \ldots, A_{n} \in \mathcal{A}$ then so is $\cup_{i=1}^{n} A_{i}$ and $\cap_{i=1}^{n} A_{i}$.

Definition 2.2 Let $\Omega$ be a set and $\mathcal{A}$ an algebra of $\Omega$. A (finitely additive) measure on $\mathcal{A}$ is a non-negative function from $\mathcal{A}$ to $\mathbb{R}$, where we write $|A|$ for the image of $A$ in $\mathcal{A}$, such that if $A, B \in \mathcal{A}$ with $A \cap B=\emptyset$ then $|A \cup B|=|A|+|B|$.

Proposition 2.3 If $\mathcal{A}$ is an algebra of a set $\Omega$ and || a measure on $\mathcal{A}$ then we have:

(i) $A, B \in \mathcal{A}$ and $A \subseteq B$ implies that $|B \backslash A|=|B|-|A|$.

(ii) If $A, B_{1}, \ldots, B_{n} \in \mathcal{A}$ and $A \in \cup_{i=1}^{n} B_{i}$ then $|A| \leq \sum_{i=1}^{n}\left|B_{i}\right|$.

Proof. We express $B$ as the disjoint union $(B \backslash A) \cup A$. As for (ii), we have pairwise disjoint sets $D_{i} \in \mathcal{A}$ with the same union as the $B_{i}$ on writing $D_{1}=B_{1}, D_{2}=B_{2} \backslash B_{1}, \ldots, D_{n}=B_{n} \backslash B_{n-1}$, thus $\left|\cup_{i=1}^{n} B_{i}\right|=\sum_{i=1}^{n}\left|D_{i}\right| \leq$ $\sum_{i=1}^{n}\left|B_{i}\right|$. Now

$$
|A|=\left|\cup_{i=1}^{n}\left(D_{i} \cap A\right)\right|=\sum_{i=1}^{n}\left|D_{i} \cap A\right| \leq \sum_{i=1}^{n}\left|D_{i}\right| .
$$

Proposition 2.4 If $G$ is any group then the collection $\mathcal{C}$ of finite unions of left cosets and right cosets of finite index subgroups of $G$ (along with $\emptyset$ ) is an algebra of $G$.

Proof. On taking $A=\cup_{i=1}^{m} C_{i}$, where $C_{i}$ is a coset (possibly left, possibly right) of the finite index subgroup $H_{i}$, we can consider $H=H_{1} \cap \ldots \cap H_{m}$ which is of finite index in $G$, and we see that each $C_{i}$ is also a finite union of (left or right, as above) cosets of $H$. Then we drop down to the core $R$ of $H$ in $G$, which is also of finite index but is normal in $G$ too. Now each $C_{i}$ is just a finite union of cosets of $R$, where $g R=R g$ for all $g \in G$. Thus 
$\cup_{i=1}^{m} C_{i}$ is just a subset of the finite quotient group $G / R$. Next if we are given $B=\cup_{i=1}^{n} D_{i}$ which is also in $\mathcal{C}$, we have $S$ normal and finite index in $G$ (for which we write $S \unlhd_{f} G$ ) with $\cup_{i=1}^{n} D_{i}$ a subset of $G / S$. But $R \cap S \unlhd_{f} G$ and $A, B$ are both subsets of $G /(R \cap S)$, meaning that $A \cup B$ and $A \backslash B$ are too.

Proposition 2.5 For any group $G$ and the algebra $\mathcal{A}$ of $G$ as defined in Proposition 2.4, let $A \in \mathcal{A}$ consist of $r$ elements of the quotient group $G / R$, where $R \unlhd_{f} G$ has index $i$. Then setting $|A|=r / i$ gives a measure on $\mathcal{A}$ with $|G|=1$.

Proof. On taking disjoint $A$ and $B$ with $A$ consisting of $r$ elements of $G / R$ and $[G: R]=i$ but $B$ equal to $s$ elements of $G / S$ with $[G: S]=j$, we have that $A$ is $r k$ elements of $G /(R \cap S)$ where $k=[R: R \cap S]$ and $B$ is $s l$ elements for $l=[S: R \cap S]$. But these subsets of $G /(R \cap S)$ must be disjoint, so $A \cup B$ is $r k+s l$ elements, giving $|A \cup B|=r k \cdot 1 / k i+s l \cdot 1 / l j=|A|+|B|$.

Clearly multiplying a measure by a strictly positive constant results in another measure. We will adopt the convention that $|G|=1$ when considering arbitrary groups, but when we are restricting ourselves purely to finite groups we assume $|A|$ is just the number of elements in $A$ for consistency of notation with finite sets.

Proposition 2.6 For any group $G$ and the algebra $\mathcal{A}$ of $G$ as above, we have $g A, A g \in \mathcal{A}$ with $|g A|=|A g|=|A|$ for all $A \in \mathcal{A}$ and $g \in G$, as well as $\left|A^{-1}\right|=|A|$. We also have for $A, B \in \mathcal{A}$ with $A \subseteq B$ that $|A|=|B|$ implies that $A=B$.

Proof. If $A$ is a subset of $G / R$ as above then the (left) action of $G$ on $G / R$ by multiplication on the left or the (right) action of multiplication on the right preserves the size of subsets. Similarly as $g R$ and $(g R)^{-1}=g^{-1} R$ are both cosets of $R$, we have $|A|=\left|A^{-1}\right|$. Moreover if $B$ is a subset of $G / S$ as above then $A$ and $B$ are both subsets of $G /(R \cap S)$ with $A$ in $B$. Therefore $A \neq B$ means that $|B| \geq|A|+|G| /[G: R \cap S]$. 
Definition 2.7 Given a group $G$, we say the basic measure $(\mathcal{B},||)$ on $G$ is the one defined in Proposition 2.5. We say that a measure $(\mathcal{A},\|\|)$ on $G$ is acceptable if it is left invariant (meaning that $g S \in \mathcal{A}$ with $\|g S\|=\|S\|$ for all $S \in \mathcal{A}$ ), or right invariant, and if it extends the basic measure, that is $\mathcal{B} \subseteq \mathcal{A}$.

Note that if $G=g_{1} H \cup \ldots \cup g_{i} H$ for $H$ a subgroup of index $i$ then $\left\|g_{1} H\right\|=\ldots=\left\|g_{i} H\right\|$ so that \|\| agrees with $\|$ on $\mathcal{B}$ up to a constant factor (if $\|||$ is not identically zero). Examples of acceptable measures extending the basic measure occur whenever $G$ is infinite amenable. Another case is that of Hartman measure, as explained in [24]: here $G$ is a topological group with a continuous homomorphism $\theta$ into a compact group $C$ (such as $G$ a finitely generated group with the profinite topology and $C$ its profinite completion). Thus $C$ has Haar measure $\mu$ and a continuity subset $S$ is a measurable subset of $C$ such that the topological boundary $\partial S$ has zero measure. We then have an algebra of subsets $A$ of $G$ consisting of the pullback $A=\theta^{-1}(S)$ of continuity subsets $S$ of $C$ and on setting the measure of $A$ to be $\mu(S)$, we see that this is well defined.

The finite residual $R_{G}$ of an infinite group $G$ is defined to be the intersection of all finite index subgroups of $G$ and we say that $G$ is residually finite if $R_{G}$ is the trivial group $I$. We have that $G / R_{G}$ is residually finite for any group $G$, so when considering the measure on $G$ as defined in Proposition 2.5, we may as well assume that $G$ is residually finite, because the measurable subsets of $G$ are just the pullback of the measurable subsets of $G / R_{G}$. Now $G / R_{G}$ may well be infinite, which is equivalent to $G$ having infinitely many subgroups of finite index, but if $G / R_{G}$ is finite then our measure is nothing other than (normalised) counting measure on $G / R_{G}$ and we are firmly in the realm of finite groups. In particular if $G$ has no proper finite index subgroups then we have a trivial algebra consisting of $G$ with $|G|=1$ and $\emptyset$ with $|\emptyset|=0$. However many infinite groups are known to be residually finite, for instance finitely generated linear groups over any field.

\section{Growth of subsets}

For $A, B$ subsets of a group $G$ with the basic measure $(\mathcal{B},||)$, we let the product set $A B=\{a b: a \in A, b \in B\}$. We have that if $A, B \in \mathcal{B}$ then so is $A B$ because we can work in $G /(R \cap S)$ as above. We are interested 
in comparing $|A B|$ with $|A|$ and $|B|$. For a finite group we certainly have $|A B| \leq|A||B|$ but in this section we are especially interested in when $|A B|$ is small. There is much on this subject, going back to Cauchy for abelian groups right up to recent interest in the non abelian case; see for instance [23] and also [22] Section 2, especially 2.7 for the non commutative case. Here we summarise the results we need, giving proofs when we generalise from finite groups to the measurable case. The first is a non abelian version of [22] Proposition 2.2.

Proposition 3.1 For $A$ and $B$ measurable non empty subsets of a group $G$ with the basic measure, we have $|A B|=|A|$ if and only if there is a finite index subgroup $H$ of $G$ such that $A$ is a union of left cosets of $H$ and $B$ is contained in a right coset of $H$. The subgroup $H$ can be taken to be $S_{R}(A)$, the stabiliser of $A$ under right multiplication of $G$.

Proof. First if $A=x_{1} H \cup \ldots \cup x_{k} H$ and $B \subseteq H y$ then $A B \subseteq A H y=A y$. Conversely for any choice $b_{0} \in B$ we have $\left|A b_{0}\right|=|A|=|A B|$ and $A b_{0} \subseteq A B$ so that $A b_{0}=A B$ by Proposition 2.6. Thus $B b_{0}^{-1}$ is in $S_{R}(A)$, which has finite index in $G$ because the orbit of $A$ consists of elements of $G /(R \cap S)$ and so is finite. Consequently $B$ is in the right coset $S_{R}(A) b_{0}$. But $a S_{R}(A) \subseteq A$ for all $a \in A$ so $A$ is a union of left cosets of $S_{R}(A)$.

We now look at Ruzsa distance, introduced in [19] for abelian groups and generalised in [23] for non abelian groups.

Definition 3.2 Let $A$ and $B$ be non empty measurable sets of a group $G$ with the basic measure. The (left) Ruzsa distance is defined as

$$
d(A, B)=\log \frac{\left|A B^{-1}\right|}{|A|^{1 / 2}|B|^{1 / 2}} .
$$

We have $\mathrm{d}(g A, g B)=\mathrm{d}(A, B)$ and $\mathrm{d}(A g, B g)=\mathrm{d}(A, B)$ too. It also satisfies the symmetric and triangle inequality, as shown in [23] Lemma 3.2, but fails the zero axiom in both directions in that we can have $\mathrm{d}(A, A)>0$ (in which case $\mathrm{d}(A, B) \geq \mathrm{d}(A, A) / 2$ for all non empty measurable $B$ ) and we can have $\mathrm{d}(A, B)=0$ but $A \neq B$, as will be seen below. Also we do not have $\mathrm{d}(A, B)$ equal to $\mathrm{d}\left(A^{-1}, B^{-1}\right)$ in general as $\left|A B^{-1}\right|$ need not equal $\left|B^{-1} A\right|$ : indeed we might not even have $\left|A A^{-1}\right|=\left|A^{-1} A\right|$. This is well known, for instance when $A=H \cup x H$ for $H$ a subgroup of $G$ and $x H x^{-1} \neq H$. Here we quantify this 
point.

Example: If $H$ is a subgroup of the finite group $G$ then $A A^{-1}=H \cup x H \cup$ $H x^{-1} \cup x H x^{-1}$ and $A^{-1} A=H \cup H x H \cup H x^{-1} H$. Thus $\left|A A^{-1}\right| \leq 4|H|-1$ but the basic theory of double cosets tells us that $|H x H|=|H|^{2} /\left|H \cap x H x^{-1}\right|$. For instance if $G$ is the symmetric group $\operatorname{Sym}(2 n)$ and $H$ is the subgroup fixing $n+1, n+2, \ldots, 2 n$ then we can take $x=(1, n+1)(2, n+2) \ldots(n, 2 n)$, so that $x H x^{-1}$ fixes $1,2, \ldots, n$ with $|H x H|=|H|^{2}$. Thus we have $A A^{-1}=4(n !)-2$ but $\left|A^{-1} A\right|=(n !)^{2}+n$ !, with $\left|A A^{-1}\right| /\left|A^{-1} A\right|$ tending to 0 as $n$ tends to infinity.

However we can get round this by defining right Ruzsa distance with $\left|B^{-1} A\right|=\left|A^{-1} B\right|$ in place of $\left|A B^{-1}\right|$. This is also left and right invariant under multiplication by group elements, and if required we can add both to get double Ruzsa distance $\operatorname{dd}(A, B)$ which will still be symmetric and satisfy the triangle inequality. The next statement for finite groups is Proposition 2.38 in [22] (with proof left as an exercise).

Proposition 3.3 We have $d(A, B)=0$ if and only if $A=g H$ and $B=\gamma H$ for $g, \gamma \in G$ and $H$ some finite index subgroup of $G$.

Proof. This implies that $|A|=|B|=\left|A B^{-1}\right|$. Therefore by Proposition 3.1 we have that there is a finite index subgroup $H$ such that $A$ is a union of left cosets of $H$ and $B^{-1}$ is contained in a right coset of $H$. This implies $|A| \geq|H| \geq|B|$ so all are equal and we must have $g, \gamma \in G$ such that $A=g H$ and $B^{-1}=H \gamma^{-1}$ so $B=\gamma H$.

Of course if $A=g H$ is a left coset of $H$ then it is also a right coset, but of $g \mathrm{Hg}^{-1}$. We make the following definition:

Definition 3.4 We say that $A$ is a left right coset in a group $G$ if it is both a left coset and a right coset of the same subgroup $H$ in $G$.

This is equivalent to saying we have some $g \in G$ for which $A=g H=H g$, which holds if and only if $g$ is in the normaliser $\operatorname{Norm}(H)$.

Corollary 3.5 We have $d(A, A)=0$ if and only if $A$ is a left coset of a finite index subgroup. Also $d\left(A, A^{-1}\right)=0$ if and only if $A$ is a left right coset of a finite index subgroup. 
Proof. The first statement is immediate from Proposition 3.3 and if $A=g H$ with $A^{-1}=\gamma H$ then $A=H \gamma^{-1}$, so $A$ is a right coset of $H$ containing $g$.

We also have similar results for the right Ruzsa distance on swapping left and right cosets. Moreover we can put these together for the double distance.

Corollary 3.6 We have $d d(A, B)=0$ if and only if there exists a finite index subgroup $H$ and $g, \gamma \in G$ with $\gamma^{-1} g \in N o r m(H)$ such that $A=g H$ and $B=\gamma H$.

Proof. We have finite index subgroups $H, L$ of $G$ with $A=g H=\left(g H g^{-1}\right) g=$ $L g_{1}$ and $B=\gamma H=\left(\gamma H \gamma^{-1}\right) \gamma=L \gamma_{1}$. Now a set can only be a right coset of two subgroups if these subgroups are equal (by taking the coset containing the identity in each case), so we have $L=g H g^{-1}=\gamma H \gamma^{-1}$, with $g H=L g$ and $\gamma H=L \gamma$.

This means that although double Ruzsa distance is not a metric, it is not so far away from being so. For instance it is on measurable subsets which are cosets of any finite index subgroup that equals its own normaliser. Also if we restrict to subgroups, we have immediately from Proposition 3.3:

Corollary 3.7 Ruzsa distance is a metric on the finite index subgroups of any group $G$.

Here it does not matter if we use left or right Ruzsa distance, which will be equal, or double Ruzsa distance which is twice each of these.

We now consider growth in groups, which has been much studied for finite subsets. The idea is to take any measurable set $A$ and consider the sizes $A, A^{2}, A^{3}$ and so on. This occurs in the theory of covering groups by conjugacy classes, which involves taking a group $G$, usually a finite simple group, and a non trivial conjugacy class $C$ so as to determine the smallest value of $n$ such that $C^{n}=G$. The monograph [2] discusses the theory in this area, and generalises $C$ to any subset of an arbitrary finite group in Chapter 3 , obtaining results not unlike those that follow.

Theorem 3.8 Given a group $G$ with the basic measure $(\mathcal{B},||)$ and non empty $A \in \mathcal{B}$, consider the sequence of measurable sets $A^{2}, A^{3}, \ldots$ We have: 
(i) There exists $n$ such that $\left|A^{n}\right|=\left|A^{n+1}\right|$ and this implies that $\left|A^{n+1}\right|=$ $\left|A^{n+2}\right|=\ldots$

(ii) We have $\left|A^{n}\right|=\left|A^{n+1}\right|$ if and only if $A^{n}$ is equal to a left right coset $g H$.

Proof. (i) If we express $A$ as a subset of $G / R$ then so is $A^{2}, A^{3}, \ldots$ so we will assume that $G$ is a finite group. As $A \neq \emptyset$, we have that $a A^{n} \subseteq A^{n+1}$ for any $a \in A$ so $\left|A^{n}\right|$ is a non decreasing sequence with values in $\{0,1 /[G: R], 2 /[G$ : $R], \ldots, 1\}$ which must eventually stabilise. But if $\left|A^{n}\right|=\left|A^{n+1}\right|$ then, on taking a particular $\alpha \in A$, we have $\left|A^{n}\right|=\left|A^{n} \alpha\right|=\left|A^{n+1}\right|$ with $A^{n} \alpha \subseteq A^{n+1}$ so $A^{n} \alpha=A^{n+1}$ by Proposition 2.6. Consequently we have $a A^{n} \subseteq A^{n} \alpha$ for all $a \in A$. Thus for $a_{1}, a_{2} \in A$ we have $a_{1} a_{2} A^{n} \subseteq a_{1} A^{n} \alpha \subseteq A^{n} \alpha^{2}$, so $A^{n+2} \subseteq A^{n} \alpha^{2}$ and $\left|A^{n+2}\right|=\left|A^{n}\right|=\left|A^{n+1}\right|$.

For (ii), first suppose that $|A|=\left|A^{2}\right|$, in which case we have that the Ruzsa distance $\mathrm{d}\left(A, A^{-1}\right)=0$. Therefore $A$ is a left right coset by Corollary 3.5. The general result follows on setting $S=A^{n}$ because $\left|A^{n}\right|=\left|A^{n+1}\right|$ implies that $\left|A^{n}\right|=\left|A^{2 n}\right|$ from (i), thus $|S|=\left|S^{2}\right|$ giving $S=g H=H g$. Conversely if $A^{n}$ is a left right coset $g H=H g$ then $A^{2 n}=g H \cdot H g=g H g=H g^{2}$ so $\left|A^{n}\right|=\left|A^{2 n}\right|$. As $\left|A^{n}\right|$ is increasing, we have $\left|A^{n}\right|=\left|A^{n+1}\right|$.

This gives us a complete answer as to which measurable subsets $A$ of $G$ "expand to become the whole group"; meaning that there exists $n>0$ such that $A^{n}=G$ (and then of course $A^{m}=G$ for all $m \geq n$ ).

Corollary 3.9 Given a non empty measurable subset $A \in \mathcal{B}$ for $G$ equipped with the basic measure $(\mathcal{B},||)$, we have that $\left|A^{n}\right|<|G|$ for all $n \in \mathbb{N}$ if and only if $A$ is a subset of a left right coset of a proper finite index subgroup $H$ of $G$.

Proof. If $A \subseteq g H=H g$ for $H$ a proper finite index subgroup then $A^{n} \subseteq g^{n} H=H g^{n}$ which is still a coset of $H$, thus never equal to $G$. Conversely if $\left|A^{n}\right|=\left|A^{n+1}\right|<|G|$, so that $A^{n}=g H=H g$ for $H$ a proper subgroup of $G$, then take any $b \in A^{n-1}$. For all $a \in A$ we have $b a \in A^{n}=g H$ so that $A$ is contained in the coset $b^{-1} g H$ which must be equal to $a H$ for any $a \in A$. Now $A^{n}=g H$ so $A^{n+1}=a g H=g H a$, meaning that (for $x=g^{-1} a g$ ) we have $x H=H a$, with $a \in x H$ so $a H=H a$. 
One might think that any generating set for $G$ expands to become all of $G$ but Corollary 3.9 illustrates a dichotomy even within finite groups in that some groups $F$ can have a set $S$ which generates $F$ but such that $S^{n}$ is a proper subset of $F$ for all $n \in \mathbb{Z}$. Certainly if $\langle S\rangle=F$ then $\cup_{n \in \mathbb{Z}} S^{n}=F$ (where we take $S^{0}=\{e\}$ ) and even $\cup_{n \in \mathbb{N}} S^{n}=F$ because $F$ is finite, so that $S^{-1} \subseteq S^{|F|-1}$. Moreover if $e \in S$ then $S^{m} \subseteq S^{n}$ so that $F=S^{N}$ for some $N>0$. But this need not be true for arbitrary generating sets if $F$ is not perfect (meaning that its commutator subgroup $F^{\prime}$ is a proper subgroup of $F$ ). For instance Theorem 4.10 of [18] claims that if $G$ is a finite abelian group and $A$ a subset that generates $G$ then there exists $N>0$ with $A^{N}=G$. In fact any finite abelian group has such an $A$ with $A^{N} \neq G$ for all $N$. The problem is the first three lines: we cannot assume without loss of generality that the identity is in $A$.

Theorem 3.10 If $F$ is a finite group then there exists a generating set $S$ for $F$ with $S^{N} \neq F$ for all $N \in \mathbb{Z}$ if and only if $F$ is not perfect. There exists a symmetrised generating set $S$ (one where $S^{-1}=S$ ) with $S^{N} \neq F$ for all $n \in \mathbb{Z}$ if and only if $F$ surjects to the cyclic group $C_{2}$.

Proof. Let $\langle S\rangle=F$ and consider the sequence $|S|,\left|S^{2}\right|,\left|S^{3}\right|, \ldots$. Either we have some $n$ with $\left|S^{n}\right|=|F|$ or some $n$ with $\left|S^{n}\right|=\left|S^{n+1}\right|<|F|$ but the latter occurs exactly when $S \subseteq g H=H g$ for $H$ a proper subgroup of $F$ by Corollary 3.9. But $S$ is a generating set contained in $\langle g, H\rangle$ so we must have $\langle g, H\rangle=F$. Now $g H g^{-1}=H$ means that $H$ is normal in $F$ with $F / H$ non trivial and cyclic. Thus if $F$ has no non trivial abelian quotients then such a generating set $S$ cannot exist, whereas if $F$ is not perfect we have $H \unlhd F$ with $F / H$ cyclic and non trivial. Thus we can take $S=g H$ where $g H$ generates $F / H$.

Now suppose that $F$ has a subgroup $H$ of index 2 then the non trivial coset $S=g H=H g$ generates $F$ and is symmetric, but $S^{n}$ is either $H$ or $g H$. Conversely if we have $S=S^{-1}$ with $\langle S\rangle=F$ but $S^{n} \neq F$ for any $n \in \mathbb{N}$ then again $S \subseteq g H=H g$ for $H$ a proper subgroup of $F$. But now $\langle g, H\rangle=F$ with $H \unlhd F$ and $S^{-1} \subseteq H g^{-1}=g^{-1} H$. As $g H \cap g^{-1} H=\emptyset$ unless $g H=g^{-1} H$, we have $g^{2} \in H$ with $[F: H]=2$.

Here is another description of the sets in Corollary 3.9.

Corollary 3.11 Given a non empty measurable subset $A$ of $G$, we have that $\left|A^{n}\right|<|G|$ for all $n \in \mathbb{N}$ if and only if $A$ is a subset of a coset of a proper 
normal subgroup of $G$, or $A$ is contained in a maximal subgroup of $G$. If $G$ is perfect then the latter always occurs.

Proof. Either $A$ is a generating set or is contained in a maximal subgroup $L$ of $G$. If the former happens then by the proof of Theorem 3.10, we have $A \subseteq g H$, where $H$ is a proper normal subgroup of $G$ and $G / H$ is cyclic.

\section{Doubling and product free sets}

If $A$ is a finite subset of a group $G$ then there has been much study on how the size of $A^{2}$ can vary with that of $A$, as we shall see later. Here we will concentrate on those results for finite groups which provide applications for measurable groups.

In [5] a subset $B$ of the finite group $G$ is called a basis for $G$ if $B^{2}=G$. The aim is then to find a basis for a given group which is as small as possible. However we will look at the two related ideas of finding subsets $A$ of $G$ which are as large as possible without being a basis for $G$, and of having a lower bound for $|A|$ which ensures that it will be a basis. The next proposition is an old and well known fact.

Proposition 4.1 If $G$ is a group of order $n$ and $A$ is a subset of $G$ with $|A|>n / 2$ then $A^{2}=G$.

Proof. For any $g \in G$ we have $\left|g A^{-1}\right|+|A|>n$ so $g A^{-1}$ and $A$ meet, giving $g \in A^{2}$.

However we will now see that this value is best possible. For groups of odd order this is straightforward.

Proposition 4.2 If $G$ is a group of order $n=2 k+1$ then we have a set $A$ of size $k$ such that $A^{2} \neq G$.

Proof. Pair off each element with its inverse, leaving out the identity $e$. On taking $A$ to be one element from each of these pairs, we have that $e \notin A^{2}$.

In fact for some very small groups $G$ it can happen that no set $A$ of size $k=(|G|-1) / 2$ or $k=|G| / 2$ (for $|G|$ odd or even respectively) has $A^{2}=G$. 
An argument in [5] shows that they always exist for $k \geq 6$. Smaller values of $k$ can then be quickly tested on computer, giving that such subsets do not exist precisely for the cyclic groups of orders 1 to 7 and abelian groups of orders 4 and 8 .

If rather than considering $\left|A^{2}\right|$ in terms of $|A|$, we consider subsets $A, B \subseteq$ $G$ and look at how $|A B|$ can depend on $|A|$ and $|B|$ then there are many known results. First note that Proposition 4.1 generalises immediately to $A B=G$ if $|A|+|B|>|G|$. Next there is the Cauchy-Davenport theorem stating that in the cyclic group $G$ of order $p$ for $p$ prime, we have $|A B| \geq$ $\min (p,|A|+|B|-1)$ for $A$ and $B$ non empty subsets of $G$. This was generalised by Kneser in 1953 to abelian groups $G$ where he showed that if $|A|+|B| \leq|G|$ then either $|A B| \geq|A|+|B|$ or $|A B|=|A|+|B|-|H|$ for $H$ a proper subgroup of $G$ and $A, B$ are both unions of cosets of $H$. In particular if $G$ is abelian and of odd order $2 k+1$ with $|A|=|B|=k$ but $|A B|<2 k$ then $|H|$ must divide $k$ so is equal to 1 , giving $|A B|=2 k-1$. In the case when $G$ is a non abelian group, Kemperman showed in [16] that for any $a \in A$ and $b \in B$, there exists a subgroup $H(a, b)$ of $G$ such that $a H b \subseteq A B$ and $|A B| \geq|A|+|B|-|H|$.

If we now look at $|A B|$ when $A$ varies over subsets of a fixed size $r$ and $B$ over size $s$ then this is covered in a series of papers by Eliahou and Kervaire. In particular if $G$ is any finite group and $1 \leq r, s \leq n=|G|$ then we can define $\mu_{G}(r, s)$ to be the minimum of $|A B|$ for $A, B \subseteq G$ with $|A|=r$ and $|B|=s$. In [7] with Plagne it was shown that

$$
\mu_{G}(r, s)=\min _{d \mid n}(\lceil r / d\rceil+\lceil s / d\rceil-1) d
$$

when $G$ is abelian. In [8] it is shown that if $G$ is solvable then for all values of $r$ and $s$ above with $r \leq s$ we have $A \subseteq B$ with $|A|=r$ and $|B|=s$ but $|A B| \leq|A|+|B|-1$. In particular if $|G|=2 k+1$ and $r=s=k$, we can invoke the Odd Order Theorem of Feit-Thompson to improve Proposition 4.2 by getting a subset $A$ of size $k$ with $\left|A^{2}\right| \leq 2 k-1$. However for general groups we cannot always take $A=B$ to obtain $\mu_{G}(r, s)$, for instance it is mentioned here that $\mu_{A l t(4)}(6,6)=9$ but $\left|A^{2}\right| \geq 10$ for $A$ a 6 element subset of Alt(4).

As for a lower bound on $\mu_{G}(k, k)$ when $|G|=2 k+1$, we have from [9] that $\mu_{G}(r, s)$ is at least the right hand side of (1) when $G$ is solvable, so for $r=s=k$ this gives us that $\mu_{G}(k, k)=2 k-1$. To complete the picture in this particular case, we have:

Proposition 4.3 If $G$ is a finite group of order $2 k+1$ and $A$ and $B$ are subsets of size $k$ then we have $|A B|=2 k-1,2 k$ or $2 k+1$ and all three values 
occur except for the cyclic group $C_{3}$ where we only obtain $2 k-1, C_{5}$ where we only obtain $2 k-1$ for $A=B$ and $2 k-1,2 k$ otherwise, and $C_{3} \times C_{3}$ where we have $2 k-1$ and $2 k+1$ for $A=B$ and all three values otherwise.

Proof. By the above results and basic calculations, we are done if we show that there exists $A \subseteq G$ with $|A|=k$ and $A^{2}$ having size exactly $2 k$ unless $G=C_{3}, C_{3} \times C_{3}$ or $C_{5}$.

We proceed by induction on $k$ and use the Odd Order Theorem to say that $G$ has a normal subgroup $N$ of order $2 r+1$, where $|G|=(2 r+1)(2 s+1)$ with $r, s \geq 1$ (unless $G$ is cyclic, in which case we can take $A=B=$ $\{1,3, \ldots, 2 k-3\} \cup\{0\})$. First assume that $N$ is not equal to any of our three troublesome cases. By induction we have $S \subseteq N$ with $|S|=r$ such that $S^{2}$ misses out precisely one element $n$ from $N$. We then take all the elements from $s$ other cosets $x_{1} N, x_{2} N, \ldots, x_{s} N$ of $N$ so that no $x_{i} N$ and $x_{i}^{-1} N$ are chosen together, as in Proposition 4.2. Placing these along with $S$ to obtain $A \subseteq G$ with $|A|=(2 r+1) s+r=k$, we have that if $a, b \in A$ with $a b \in N$ then $a, b \in S$ so $A^{2} \cap N$ is also all of $N$ except $n$. However if we have a coset $y N$ with $y \notin N$ then there are two elements $c N, d N$ from the set $\left\{N, x_{1} N, \ldots, x_{s} N\right\}$ of size $s+1$ with $c N d N=y N$ by Proposition 4.1. Let us define subsets $C, D$ of $A$ where $C=c N$ and $D=d N$, unless $c$ (or $d$ ) is the identity $e$ in which case we let $C$ (or $D$ ) be equal to $S$. However $c$ and $d$ are not both $e$, so $|C|+|D|>|N|$ and hence $C D$ is all of $y N$ (by considering $d^{-1} c^{-1} C d$ and $d^{-1} D$ as subsets of $N$ ).

If however $N$ is troublesome then we can form $A$ as before but then we find a non trivial coset $x N$ and remove $x$ from $A$, so that $|A \cap x N|=2 r$. We then select just the one element $x^{-1}$ from its inverse $x^{-1} N$ in $G / N$ and put this back in $A$. Provided that $r \geq 2$ the argument works as before because $|A \cap x N|+|A \cap N|=3 r>2 r+1$, and the elements of $N$ in $A^{2}$ can only come from $(x N \cap A) x^{-1}, x^{-1}(x N \cap A)$ and $(A \cap N)(A \cap N)$. But the first two sets are equal to $N-\{e\}$ so we can choose $A \cap N$ to consist of $r$ elements such that $(A \cap N)(A \cap N)$ misses $e$ by Proposition 4.2.

Now suppose that $r=1$, so that $N=C_{3}$. This argument will still ensure that $A^{2} \cap N=N-\{e\}$ but now we have $|A \cap x N|=2$ and $|A \cap N|=1$, meaning that $A^{2}$ need not cover all of $x N$. However if we can find $x$ with $x N$ having order greater than 3 in $G / N$ then we can ensure that we choose all of $x^{2} N$ as one of our cosets which are fully contained in $A$, thus $x N=x^{-1} \cdot x^{2} N$ is in $A^{2}$.

Finally if $G / N$ has more than 5 elements then we can find $a N$ and $b N$ 
disjoint from all of $N, x N$ and $x^{-1} N$ with $a N b N=x N$, so we include all of $a N$ and $b N$ in $A$ too, leaving only $|N|=|G / N|=3$.

Now we move to groups of even order where it is clear that we can have $H^{2}=H$ with $|H|=|G| / 2$ if $H$ is a subgroup of index 2 in $G$, or a coset thereof. Indeed if a subset $S$ of a finite group $G$ satisfies $\left|S^{2}\right|=|S|$ then we already know that $S$ must be a left right coset by Theorem 3.8 (ii). However there certainly are many groups of even order possessing no subgroup of index 2: for instance the alternating groups $\operatorname{Alt}(n)$ for $n \geq 4$. If $r+s=|G|$ then we can easily find $A$ and $B$ of sizes $r$ and $s$ with $|A B| \neq G$ by setting $B$ equal to the complement of $A^{-1}$, but we would like to take $A=B$.

The following applies to all groups of even order without needing any knowledge of finite simple groups.

Theorem 4.4 If $G$ has order $2 k$ then there exists a subset $S$ of size $k$ with $S^{2} \neq G$.

Proof. The requirement that there is a $g \in G$ with $g \notin S^{2}$ is equivalent to $S \cap g S^{-1}$ being empty. We will try to find a suitable $g$ and a symmetric $S$, so that it is enough to confirm $S \cap g S=\emptyset$.

We assume that $g$ is of even order: note that this ensures that $g^{i}$ is never conjugate to $g^{j}$ if $i$ and $j$ are of different parities. We further ask that $g^{i}$ is never a square in $G$ when $i$ is odd. This can be guaranteed by taking $g$ to have order $m=2^{r} l$ where $l$ is odd and $r$ is as large as possible amongst the elements of $G$.

We now consider the action of left multiplication by $g$ on $G$. This is a free action so each orbit is a $m$-cycle and there are $2 k / m$ of them. On taking $\operatorname{Orb}(x)$ for $x \in G$, the idea is to form $S$ and its complement $T=g S$ by placing $g^{i} x$ in $S$ for $i$ even and $T$ for $i$ odd. However we need to ensure that inverses are placed together. If $x^{-1} \in \operatorname{Orb}(x)$ then this is fine as $g^{i}$ cannot equal $x^{-2}$ for $i$ odd. However in general $\operatorname{Orb}\left(x^{-1}\right)$ will be a separate orbit and hence disjoint from $\operatorname{Orb}(x)$. To achieve this, we consider a different action on $G$ by the group $C_{m} \times C_{2}$ where the action of the first component is conjugation by $g$ and the second sends $x$ to $x^{-1}$ : these do commute. We then place the orbits under the new action of $x, g^{2} x, \ldots, g^{m-2} x$ in $S$ and those of $g x, g^{3} x, \ldots, g^{m-1} x$ in $T$. Note that we are not claiming these are all distinct orbits or even that the orbits are of the same size, but this is a well defined procedure unless the orbits of $g^{i} x$ and $g^{j} x$ are equal for $i$ odd and $j$ even. 
However then we would have some $k$ such that $g^{i} x$ is equal to $g^{k+j} x^{ \pm 1} g^{-k}$. The plus sign implies that $x$ conjugates $g^{-k}$ to $g^{i-k-j}$, with the two indices having different parities, so different orders, whereas taking the minus sign means that $\left(g^{k} x\right)^{2}=g^{2 k+j-i}$ but $2 k+i-j$ is odd so this is not a square.

Having chosen $x$, we have now partitioned all elements of the form $g^{i} x^{ \pm 1} g^{j}$ for arbitrary $i$ and $j$, so we now choose any $y$ not in this set and start again. We now need to show that for any $\gamma \in G$ we have that $\gamma^{-1}$ is in the same subset and $g \gamma$ is not. This ensures that $S \cap T=\emptyset$ with $T=g S$, and as $S \cup T=G$ we obtain $|S|=k$. We express $\gamma$ as $g^{i} z^{ \pm 1} g^{j}$ where $z \in G$ was one of the elements picked out during the above process. We see that $\gamma$ is in the orbit of $g^{i+j} z$ or $g^{-i-j} z$, so certainly $\gamma^{-1}$ is in the same orbit and $g \gamma$ will be in the orbit of $g^{i+j+1} z$ or $g^{-i-j-1} z$ respectively, either of which end up on the other side.

Corollary 4.5 There exist finite simple groups $G$ and subsets $S$ of $G$ with $|S|=|G| / 2$ such that $|G|-\left|S^{2}\right|$ can be arbitrarily large.

Proof. The above construction showing that $g \notin S^{2}$ also shows that $g^{i} \notin S^{2}$ for odd $i$, so we miss out on at least $m / 2$ elements where $m$ is the order of $g$. Thus we merely require finite simple groups with elements of order an arbitrarily high power of 2 , and even the alternating groups provide this.

Other applications of these results enable us to give counterexamples to Freiman type statements with constant 2. The original theorem, due to Freiman himself in [10], is that a subset $A$ of $G$ satisfies $\left|A^{2}\right|<(3 / 2)|A|$ if and only if $A$ is contained in a left right coset $g H=H g$ (where we can of course take $g$ to be any element of $A$, so that $A$ is in the normaliser of $H$ ) with $|A|>2 / 3|H|$ and such that $A^{2}$ is exactly equal to $g^{2} H=H g^{2}$. A neat proof is also given in the Tao blog of November 2009. In [4] it is asked how one can characterise sets $A$ with $\left|A^{2}\right|<2|A|$, but it is clear that the conclusion of Freiman's theorem can no longer apply: if $A=\{e, g\}$ with $A^{2}=\left\{e, g, g^{2}\right\}$ and $g$ has order at least 4 then $A^{2}$ is not a coset, because it contains $e$ and so would have to be a subgroup. In fact even if all elements of $G$ have order 1,2 or 3 the conclusion is still false:

Proposition 4.6 If $G$ is any finite group, except for a cyclic group of order 
at most 3 or $C_{2} \times C_{2}$, then there exists $A \subseteq G$ with $\left|A^{2}\right| \leq(7 / 4)|A|$ but such that $A^{2}$ is not a coset of any subgroup of $G$.

Proof. We can assume that the order of any non identity element in $G$ is 2 or 3. If $G$ has elements of order 3 then let $H_{3}$ be a Sylow 3-subgroup of $G$. We certainly have $|A|=4$ but $\left|A^{2}\right|=7$ inside $C_{3} \times C_{3}$ and $H_{3}$ surjects to this unless $H_{3}$ is cyclic, in which case it can only have order 3. Otherwise we pull back $A$ from $C_{3} \times C_{3}$ to $H_{3}$, so that $A$ is a subgroup of $G$ consisting of 4 cosets of $H_{3}$ with $A^{2}$ equal to 7 cosets out of 9 . Then $\left|A^{2}\right| /|G|$ is $7 / 9$ times a power of 2 , so $A^{2}$ is not a coset in $G$.

Similarly if a Sylow 2-subgroup $H_{2}$ of $G$ is non trivial then it must be $C_{2}$, $C_{2} \times C_{2}$ or it contains $C_{2} \times C_{2} \times C_{2}$ which has a subset $A$ of size 4 such that $\left|A^{2}\right|=7$. This leaves us with $H_{3}=C_{3}$ and $H_{2}=C_{2}$ or $C_{2} \times C_{2}$, so we are just left with $S_{3}$ and $A_{4}$ where we can have in each case $|A|=|G| / 2$ with $\left|A^{2}\right|=(5 / 3)|A|$.

We note that $7 / 4$ is best possible here even if we exclude finitely many groups as it was shown in [12] that for $A \subseteq\left(C_{2}\right)^{n}$ with $\left|A^{2}\right|<2|A|$ then either $A^{2}$ is a subgroup or $\left|A^{2}\right| \geq 7 / 4|A|$.

Consequently work on extending Freiman's (3/2) Theorem has concentrated on taking $3 / 2 \leq K<2$ and trying to show that there exists a constant $C(K)$ such that if $A$ is a subset of any finite group $G$ with $|A| \leq K\left|A^{2}\right|$ then there exists some subgroup $H$ of $G$ such that $A^{2}$ is the union of no more than $C(K)$ right (say) cosets of $H$. This cannot hold for all finite groups $G$ when $K=2$ because we can take long arithmetic progressions; namely $A=\left\{e, g, \ldots, g^{n-1}\right\}$ for $g$ an element of some group with the order of $g$ being at least $2 n$. It clearly does hold if we restrict to only finitely many groups and, using a major group theory result, we can show here that this is necessary.

Proposition 4.7 If $\mathcal{G}$ is any infinite family of finite groups then for any $C>0$ there exists a group $G$ in $\mathcal{G}$ and a subset $A$ of $G$ such that $\left|A^{2}\right|<2|A|$ but $A^{2}$ is not the union of at most $C$ right cosets of some subgroup of $G$.

Proof. We are done by long arithmetic progressions unless there is an upper bound on the exponents of all groups in $\mathcal{G}$. Moreover if there are infinitely many groups in $\mathcal{G}$ of odd order then we are also done by Proposition 4.3, because if $|G|=2 k+1$ we have $A$ with $|A|=k$ but $\left|A^{2}\right|=2 k$ or $2 k-1$, so 
that if $A^{2}$ is a union of right cosets of $H \leq G$ then $|H|$ divides $|G|$ and $\left|A^{2}\right|$. But here we can let $k$ tend to infinity.

Furthermore we are done if the odd part of the orders of the groups $G$ in $\mathcal{G}$ is unbounded, as we can take Sylow subgroups $S$ of $G$. On applying the above to $A$ in $S$, if $A^{2} \subseteq S$ is a union of right cosets of a subgroup $H$ of $G$ then these right cosets are in $S$, so $H$ is a subgroup of $S$ and the previous argument applies.

We can now restrict $\mathcal{G}$ to an infinite sequence $\left(G_{n}\right)$ of 2 -groups with bounded exponent. By Zelmanov's solution of the restricted Burnside problem, the minimum number of generators $d(n)$ of $G_{n}$ must tend to infinity with $n$, so $G_{n}$ surjects to $\left(C_{2}\right)^{d(n)}$ using the Frattini subgroup. Now in $\left(C_{2}\right)^{d}$ we have a subset $B$ of size $2^{d-1}$ with $B^{2}=2^{d}-1$; for instance take $B$ to be the set with final coefficient 0 but replacing the element $(1,1, \ldots, 1,0)$ with $(0, \ldots, 0,1)$. We then pull $B$ back to a union $A$ of cosets in $G_{n}$ of the kernel $K$ of this surjection. We obtain $\left|A^{2}\right|=\left(2-\left(1 / 2^{d-1}\right)\right)|A|$ and $\left|A^{2}\right|=\left(2^{d}-1\right)|K|$. Thus if $A^{2}$ is a union of cosets of some $H \leq G_{n}$ then suppose $\left|G_{n}\right|=2^{f(n)}$. We have $|K|=2^{f(n)-d(n)}$ and $|H|$ divides $\left|G_{n}\right|$ and $\left|A^{2}\right|$, thus $|H|$ divides $2^{f(n)-d(n)}$ meaning that $A^{2}$ would be at least $2^{d}-1$ cosets of $H$.

A recent result on product growth was established by Babai, Nikolov and Pyber in [3], following work of Gowers. This states that for a finite group $G$ of order $n$, let $m$ be the minimum degree of a non trivial (real) representation of $G$ into $G L(n, \mathbb{R})$. Then for $A, B$ non empty subsets of $G$ with $|A|=r$ and $|B|=s$ we have $|A B|>\frac{n}{1+\left(n^{2} / m r s\right)}$, with the right hand side at least the minimum of $n / 2$ and $m r s /(2 n)$. If $r \geq s$ we already have the bound that $|A B| \geq r$, but $\frac{n}{1+\left(n^{2} / m r s\right)} \geq r$ implies that $0 \geq(n-m r / 2)^{2}+r^{2}\left(m-m^{2} / 4\right)$. Thus we obtain nothing new from this inequality if $m \leq 4$, which is the case for all finite soluble groups for instance. However this inequality really comes into play as $m$ increases. In particular for finite simple groups $G$ we have that $m$ tends to infinity with the order of $G$, so this shows that although $|G|-\left|S^{2}\right|$ in Corollary 4.5 can be arbitrarily high, we must have $|G| /\left|S^{2}\right|$ tending to 1 .

This result also solves a question in [8, which asks at the start of Section 3 whether the small sumsets property holds for all finite groups $G$, that is whether for all $1 \leq r, s \leq n=|G|$ we have subsets $A, B$ of $G$ with $|A|=r$, $|B|=s$ and $|A B| \leq r+s-1$. We can find plenty of counterexamples by taking 
$\lambda$ and $\mu$ such that $|A|=\lambda n,|B|=\mu n$ and assuming that $\lambda+\mu \leq 1 / 2$. Then we would have $|A|+|B|=(\lambda+\mu) n$ but $|A B|$ is greater than the minimum of $n / 2$ and $m n \lambda \mu / 2$, so that if $m \geq 2(\lambda+\mu) /(\lambda \mu)$ then $|A B|>|A|+|B|$. Thus we obtain lots of pairs $(r, s)$ where the small sumsets property fails by taking $\lambda$ and $\mu$ as above, finding a group $G$ such that such that $m \geq 2(\lambda+\mu) /(\lambda \mu)$ and setting $(r, s)=(\lambda|G|, \mu|G|)$.

We now consider these results in the context of infinite measurable groups $G$ with $|G|=1$. For such a $G$, let $u$ be the supremum of $|A|$ over measurable sets $A$ with $A^{2}$ also measurable and such that $\left|A^{2}\right|<1$. From Proposition 4.2 and Theorem 4.4 we immediately have:

Corollary 4.8 For $G$ any group equipped with an acceptable measure and with $G / R_{G}$ infinite, we have $u=1 / 2$ and this is attained if there exists a finite quotient of $G$ with even order.

We remark that there do exist interesting examples of infinite residually finite groups where every finite quotient has odd order. For instance infinite residually finite $p$-groups (for $p \neq 2$ ) have this property. In [17] Section 5.4, a finitely generated group is said to be of prosoluble type if the group is residually finite and all finite quotients are soluble. By the Odd Order Theorem any example of a finitely generated residually finite group where all finite quotients have odd order is of prosoluble type. However such a group cannot be linear in characteristic 0 if infinite, by [17] Window 9 Corollary 17 so we can say that any infinite finitely generated linear group in characteristic 0 has a subset which is, by any reasonable definition, half of the group but whose square is not the whole group, indeed the complement of the squared set is not negligible. We also remark that all the non trivial finite quotients of the groups in Theorem 4.11 are of even order, but none are a power of 2 .

Similarly we can look at product free subsets. These are subsets $S$ of a group $G$ such that $S \cap S^{2}$ is empty. This definition makes sense for any subset in any group but a well studied problem in finite groups is to examine how big a product free subset can be. Therefore we can ask the same question of infinite groups with an acceptable measure, where we restrict $S$ to being a non empty measurable subset.

The survey article [14] is a very readable introduction to the subject in the case of finite groups and the author provides an update in [15]. We begin by noting that the obvious upper bound of half the group in the finite case for a product free subset extends immediately to the infinite measurable case. 
Proposition 4.9 If $G$ is a group with an acceptable measure and $S$ is a measurable product free subset then $|S| \leq 1 / 2$. If $G$ has the basic measure then we obtain equality if and only if $S=g H$ for $H$ a subgroup of index 2 and $g \notin H$.

Proof. For any $s \in S$ we have that $S^{2}$ contains the measurable set $s S$ with $|s S|=|S|$, so if $|S|>1 / 2$ then $S$ and $s S$ must meet. Now suppose $G$ has the basic measure and $S$ is product free with $|S|=1 / 2$, thus forcing $\left|S^{2}\right|=1 / 2$. We can then apply Theorem 3.8 (ii).

Another useful point is Observation 1 in [14, which is that if $N$ is a normal subgroup of $G$ then we can pull back a product free subset of $G / N$ to one for $G$. This also works for infinite measurable groups and the measures of the product free subsets will be the same. Therefore given any infinite group $G$ with an acceptable measure, we can get a product free subset of $G$ by looking at its finite quotients, so it is enough to concentrate on finite abelian and finite simple groups.

For any measurable group $G$ with $|G|=1$ we let $\alpha(G)$ be the supremum of $|S|$ over all product free subsets $S$ for $G$. It is shown in [1] that $\alpha(G) \geq 2 / 7$ for all finite abelian groups. Therefore we immediately have $\alpha(G) \geq 2 / 7$ for all infinite finitely generated groups which are not perfect, because there will be a non trivial finite abelian quotient. However in the case of finite simple groups it was recently shown by Gowers in [11] that $\alpha(G)$ can be arbitrarily small. Indeed using the above result of Babai, Nikolov and Pyber, which was influenced by the Gowers paper, we have that if $A=B$ with size $r$ then $\left|A^{2}\right|>\frac{n}{1+\left(n^{2} / m r^{2}\right)}$, so if the right hand side is at least $n-r$ then $A^{2}$ and $A$ would have to intersect, meaning that no product free set can be of size $r$. The latter condition is equivalent to $\lambda^{3} /(1-\lambda) \geq 1 / m$ where $\lambda=r / n$ so we see that as $m$ tends to infinity we have $\alpha(G) \rightarrow 0$. In fact this gives a complete answer to the smallest size of product free sets for any infinite family of finite groups.

Proposition 4.10 Suppose that $G_{n}$ is a sequence of non trivial finite groups then we have that the infimum of $\alpha\left(G_{n}\right)$ is non zero if and only the minimum degree $m\left(G_{n}\right)$ of a non trivial real representation is bounded.

Proof. If $m\left(G_{n}\right)$ is unbounded then we can apply the result of Babai, Nikolov and Pyber. However if $m\left(G_{n}\right)$ is bounded above by $N$ then every $G_{n}$ has a non trivial image $Q_{n}$ in $G L(N, \mathbb{R})$. By Jordan's Theorem there exists $j$ 
depending only on $N$ such that $Q_{n}$ has an abelian subgroup $H_{n}$ of index at most $j$. Either $H_{n}$ is trivial so that $1<\left|Q_{n}\right| \leq j$, or we have a product free subset $S_{n}$ of $H_{n}$, so also of $Q_{n}$ with $\left|S_{n}\right| /\left|Q_{n}\right| \geq 2 /(7 j)$. In both cases we pull the product free subset of $Q_{n}$ back to $G_{n}$.

We can answer the equivalent question for infinite measurable groups by using the construction in [13].

Theorem 4.11 There exist infinite finitely generated residually finite groups equipped with the basic measure such that $\alpha(G)$ is arbitrarily small.

Proof. It is shown in Theorem 4 of [13] that if $\Gamma=\prod_{n=1}^{\infty} S_{n}$ is an unrestricted direct product of finite simple groups $S_{n}$ with $l\left(S_{n}\right) \rightarrow \infty$, where $l\left(S_{n}\right)$ is the largest integer $l$ such that $S_{n}$ contains the alternating group $A l t(l)$, then $\Gamma$ is the profinite completion $\hat{G}$ of a finitely generated residually finite group $G$. We suppose that all $S_{n}$ are distinct and let $\Delta$ be a finite index normal open subgroup of $\Gamma$. Then for each $n$ we must have that $\Delta \cap S_{n}$ is equal to $S_{n}$ or $I$, but $\Delta \cap S_{n}=I$ means that $S_{n}$ is a subgroup of the finite group $\Gamma / \Delta$, thus this can only happen for finitely many $n$. As $S_{n}$ is in $\Delta$ for all $n>N$ say and $\Delta$ is closed, we have that there is a surjective homomorphism $\theta$ from $S_{1} \times \ldots \times S_{N}$ to $\Gamma / \Delta$. However this surjective image of a finite direct product of distinct finite simple groups can only be $S_{i_{1}} \times \ldots \times S_{i_{k}}$ for $1 \leq i_{1}<\ldots<i_{k} \leq N$ where $\operatorname{ker}(\theta) \cap S_{i_{j}}=I$ and $\operatorname{ker}(\theta) \cap S_{m}=S_{m}$ otherwise. This is because $\theta\left(S_{m}\right)$ is normal in in $\theta\left(S_{1} \times \ldots \times S_{N}\right)$, so for $m \neq n$ we have $\theta\left(S_{m}\right) \cap \theta\left(S_{n}\right)$ is normal in $\theta\left(S_{m}\right)$ and $\theta\left(S_{n}\right)$, implying that $\theta\left(S_{m}\right) \cap \theta\left(S_{n}\right)=I$ and $\theta\left(S_{1} \times \ldots \times S_{N}\right) \cong \theta\left(S_{1}\right) \times \ldots \times \theta\left(S_{N}\right)$.

Now suppose that $G$ is the finitely generated residually finite group with $\hat{G}=\Gamma$ as above. As any (continuous) homomorphism from $G$ onto a finite group $F$ extends continuously to $\Gamma$, we have that $F$ must be of the form $S_{i_{1}} \times \ldots \times S_{i_{k}}$ too. We now take any integer $K>0$ and apply Theorem 4 of [13] with $\Gamma_{K}=\Pi_{n=K}^{\infty} A l t(n)$ where certainly $l(A l t(n)) \rightarrow \infty$ as $n$ does. Then the only finite quotients $F$ of the finitely generated residually finite group $G_{K}$ are direct products of alternating groups of rank at least $K$, but we have $m(\operatorname{Alt}(n)) \geq n-1$ for $n \geq 7$ so $\alpha^{3}(\operatorname{Alt}(n)) \leq 1 / m(\operatorname{Alt}(n)) \leq 1 /(K-1)$ for $n \geq K$. This implies that $\alpha(F) \leq 1 /(K-1)^{1 / 3}$ too, because if $F$ had a non trivial representation of dimension less than $K-1$ then this would be non trivial on one of the direct factors. Consequently $\alpha\left(G_{K}\right) \leq 1 /(K-1)^{1 / 3}$ and this tends to 0 as $K$ tends to infinity. 


\section{Ruzsa distance on finite index subgroups}

Given any group $G$, we have from Corollary 3.7 that Ruzsa distance is a genuine metric on the set $\mathcal{S}(G)$ of finite index subgroups of $G$. Here we will use the double distance formula, so that for subgroups $A$ and $B$ of $G$ we have

$$
\mathrm{d}(A, B)=\log \frac{\left|A B^{-1}\right|}{|A|^{1 / 2}|B|^{1 / 2}}+\log \frac{\left|B A^{-1}\right|}{|A|^{1 / 2}|B|^{1 / 2}}=\log \frac{[G: A][G: B]}{[G: A B]^{2}} .
$$

In order to avoid countless mentions of logs, we will consider the multiplicative version of Ruzsa distance; that is

$$
\mathrm{e}(A, B)=\exp ^{\mathrm{d}(A, B)}=\frac{[G: A][G: B]}{[G: A B]^{2}}
$$

with e satisfying $\mathrm{e}(A, B)=\mathrm{e}(B, A), \mathrm{e}(A, B) \geq 1$ with equality if and only if $A=B$, and $\mathrm{e}(A, B) \leq \mathrm{e}(A, C) \mathrm{e}(C, B)$ for any $C \leq_{f} G$.

Now $A B$ need not be a subgroup but we can define the "index" $[G: A B]$ of $A B$ in $G$; if $A B$ consists of $k$ cosets of $A \cap B$ in $G$ then $[G: A B]=k /[G$ : $A \cap B]$. This need not be an integer (consider 2-cycles in the symmetric group $S_{3}$ for instance), so that it is not clear from this formula that $\mathrm{e}(A, B) \in \mathbb{N}$ but we have that $[A: A \cap B]=[A B: B]$, because if $A B$ is a union of $j$ disjoint cosets $a_{1} B, \ldots, a_{j} B$ then $a_{1}(A \cap B), \ldots, a_{j}(A \cap B)$ are disjoint cosets with union $A$. Thus we also have

$$
\mathrm{e}(A, B)=\frac{[G: A \cap B]^{2}}{[G: A][G: B]}=[A: A \cap B][B: A \cap B] .
$$

Proposition 5.1 The metric d induces the discrete topology on $\mathcal{S}(G)$.

Proof. We have that $\mathrm{d}(A, B)$ takes on values in $\log (\mathbb{N})$ so any two distinct points are at least $\log 2$ apart.

We also need to see when the triangle inequality becomes equality.

Proposition 5.2 If $A, B, C \leq_{f} G$ then we have $e(A, C) e(C, B)=e(A, B)$ if and only if $C$ contains the subgroup $A \cap B$ and $C$ is equal to $(C \cap A)(C \cap B)$. 


\section{Proof.}

We would have

$$
[A: A \cap C][C: A \cap C][B: B \cap C][C: B \cap C]=[A: A \cap B][B: A \cap B] .
$$

Now let $X=A \cap B \cap C$ and let us multiply top and bottom of the left hand side by $[Y \cap Z: X]$ for the various intersections $Y \cap Z$ of subgroups that appear on this side, and the same for the right. This gives us

$$
[C: X]^{2} /\left([A \cap C: X]^{2}[B \cap C: X]^{2}\right)=1 /[A \cap B: X]^{2}
$$

so we can take square roots to get $[C: X][A \cap B: X]=[C \cap A: X][C \cap B: X]$. The right hand side is equal to $[(C \cap A)(C \cap B): X]$ and the left hand side gives us $[C(A \cap B): X]$. But $C$ is always contained in $C(A \cap B)$ and itself contains $(C \cap A)(C \cap B)$ so all three must be equal.

Note that if $A \leq C \leq B$ for $A$ (and $B, C$ ) finite index subgroups of $G$ then $\mathrm{d}(A, C)+\mathrm{d}(C, B)=\mathrm{d}(A, B)$.

We can turn $\mathcal{S}(G)$ into a graph with a natural metric by joining the finite index subgroups $A$ to $B$ with an edge if $B$ is a maximal subgroup of $A$. We then regard the edge as an isometric copy of the interval $[0, \mathrm{~d}(A, B)]$. This gives us

Proposition 5.3 For any finite index subgroups $A$ and $B$ there exists a straight line in $\mathcal{S}(G)$ between $A$ and $B$ - that is an isometry $f:[0, d(A, B)] \rightarrow$ $\mathcal{S}(G)$ with $f(0)=A$ and $f(d(A, B))=B$.

Proof. We have subgroups $H_{0}, H_{1}, \ldots, H_{n}$ such that $A \cap B=H_{0}<H_{1}<$ $\ldots<H_{n}=A$ and we can take this to be a maximal chain because each subgroup has finite index in the one above. This means we have edges joining $H_{0}$ to $H_{1}, \ldots, H_{n-1}$ to $H_{n}$ and as $\mathrm{d}(A \cap B, A)=\mathrm{d}\left(H_{0}, H_{1}\right)+\ldots+\mathrm{d}\left(H_{n-1}, H_{n}\right)$ by Proposition 5.2, we have that the join of these edges has length $\mathrm{d}(A, A \cap B)$. We can do the same with $B$ in place of $A$ and then put these two joins together, with $\mathrm{d}(A, A \cap B)+\mathrm{d}(A \cap B, B)$ also equal to $\mathrm{d}(A, B)$ by Proposition 5.2 .

However the mere existence of a graph possessing a metric and with vertices in bijective correspondence with the finite index subgroups of a group 
$G$ is not special unless we can say something about how $G$ acts. We would like an isometric action; even better would be a faithful isometric action. The first is easy to ensure.

Proposition 5.4 The group $G$ acts by conjugation on the graph $\mathcal{S}(G)$ and this action is isometric.

Proof. We have that if $H \leq_{f} G$ then $g H g^{-1}$ is a subgroup of the same index, and if $L$ is a maximal subgroup of $H$ then $g L^{-1}$ is maximal in $g H^{-1}$ so they are still joined by an edge of the same length.

However there are two cases where it is clear that we will have elements acting trivially: the first is that any element in the finite residual $R_{G}$ would also be in $N(H)$ for any $H \leq_{f} G$, and hence in the kernel $K(G)$ of our action. Therefore we will say without loss of generality that $G$ is residually finite by replacing it with $G / R_{G}$, whereupon the metric space $\mathcal{S}\left(G / R_{G}\right)$ is equal to $\mathcal{S}(G)$. The second case is when $G$ has a non trivial centre $Z(G)$ which will act trivially, so the whole action is trivial if $G$ is an abelian group. It would be good if $K(G)$, which is the intersection of the normalisers $N(H)$ over all finite index subgroups $H$, were equal to the centre when $G$ is residually finite, but a moment's reflection on finite groups reminds us that every subgroup of the (non abelian) Quaternion group of order 8 is normal, so here too is a trivial action.

In fact this hope is not too far off the truth. For although we have not seen the group $K(G)$ in the literature, there is a very similar concept which reduces to $K(G)$ when $G$ is finite. The Baer norm $B(G)$ is defined to be the intersection $N(H)$ of normalisers over all subgroups $H$ of $G$. The crucial fact is in 21] from 1960: that for any group $G$ the Baer norm $B(G)$ is in the second centre $Z_{2}(G)$, which is formed by quotienting $G$ by its centre and taking the pullback of the centre of this quotient $G / Z(G)$ to get $Z_{2}(G)$.

This has an immediate application to our situation:

Proposition 5.5 Let $G$ be a group which is residually finite. Then the kernel $K(G)$ of our action is contained in $Z_{2}(G)$. In particular the action is faithful if and only if $G$ is residually finite with trivial centre.

Proof. Given $g$ in $G$, if $g \notin Z_{2}(G)$ then there exists $x \in G$ such that the commutator $[g, x]=g x g^{-1} x^{-1}$ is non trivial in $G / Z(G)$. Thus there further 
exists some $y \in G$ such that the element $z=[[g, x], y] \neq e$ in $G$. Now take a finite quotient $Q$ of $G$ with (the image of) $z$ non trivial in $Q$. If $g$ were in the second centre $Z_{2}(Q)$ then $[g, x] Q$ is in the centre of $Q$, a contradiction. Thus $g Q \notin B(Q)$, giving a subgroup $S$ of $Q$ with $g S g^{-1} \neq S$. Now pull back to obtain a finite index subgroup $H$ of $G$ with $g H^{-1} \neq H$.

The last part of Proposition 5.5 is relying on the fact that if $g \in K(G)$ and $Q$ is any quotient of $G$ then (the image of) $g$ is in $K(Q)$ too by the correspondence theorem. Thus for residually finite groups $G$ we have $B(G)$ is trivial if and only if $K(G)$ is trivial too, which occurs if and only if $G$ has trivial centre.

Corollary 5.6 If $G$ is a finitely generated group then we have a faithful isometric action of $G / K(G)$ on the connected graph $\mathcal{S}(G)$ such that every point has a finite orbit. Moreover $G / K(G)$ is residually finite.

Proof. The first part follows from the results of this section, along with the well known fact that a finitely generated group has only finitely many subgroups of a given finite index. As $Q=G / K(G)$ acts faithfully on $\mathcal{S}(G)$, if $q$ is not the identity in $Q$ then we have $s \in \mathcal{S}(G)$ with $q(s) \neq s$. But as the orbit of $s$ is finite, the stabiliser of $s$ is a finite index subgroup of $Q$ missing $q$.

\section{References}

[1] N. Alon and D. J. Kleitman, Sum-free subsets. A tribute to Paul Erdös, 13-26, Cambridge Univ. Press, Cambridge, 1990.

[2] Z. Arad, J.Stavi and M. Herzog, Powers and products of conjugacy classes in groups. Products of conjugacy classes in groups, 6-51, Lecture Notes in Math. 1112, Springer, Berlin, 1985.

[3] L. Babai, N. Nikolov and L. Pyber, Product growth and mixing in finite groups. Proceedings of the Nineteenth Annual ACM-SIAM Symposium on Discrete Algorithms, 248-257, ACM, New York, 2008. 
[4] Y. Berkovich, Questions on set squaring in groups. Structure theory of set addition, Asterique 258 (1999), 249-253.

[5] E. A. Bertram and M. Herzog, On medium-size subgroups and bases of finite groups, J. Combin. Theory Ser. A 57 (1991), 1-14.

[6] K. J. Böröczky, P. P. Pálfy and O. Serra, On the cardinality of sumsets in torsion-free groups, http://arxiv.org/abs/1009.6140 (2010)

[7] S. Eliahou, M. Kervaire and A. Plagne, Optimally small sumsets in finite abelian groups, J. Number Theory 101 (2003), 338-348.

[8] S. Eliahou and M. Kervaire, The small sumsets property for solvable finite groups, European J. Combin. 27 (2006), 1102-1110.

[9] S. Eliahou and M. Kervaire, Minimal sumsets in finite solvable groups, Discrete Math. 310 (2010), 471-479.

[10] G. A. Freiman, Groups and the inverse problems of additive number theory (Russian). Number-theoretic studies in the Markov spectrum and in the structural theory of set addition, 148-162. Kalinin. Gos. Univ., Moscow, 1973.

[11] W.T. Gowers, Quasirandom groups, Combin. Probab. Comput. 17 (2008), 363-387.

[12] F. Hennecart and A. Plagne, On the subgroup generated by a small doubling binary set, European J. Combin. 24 (2003), 5-14.

[13] M. Kassabov and N. Nikolov, Cartesian products as profinite completions, Int. Math. Res. Not. (2006), Art. ID 72947, 17pp.

[14] K. S. Kedlaya, Product-free subsets of groups, Amer. Math. Monthly 105 (1998), 900-906.

[15] K.S. Kedlaya, Product-free subsets of groups, then and now. Communicating mathematics, 169-177, Contemp. Math. 479, Amer. Math. Soc., Providence, RI, 2009.

[16] J.H. B. Kemperman, On complexes in a semigroup, Indag. Math. 18 (1956), 247-254. 
[17] A. Lubotzky and D. Segal, Subgroup growth. Progress in Mathematics 212, Birkhaüser Verlag, Base, 2003.

[18] M. B. Nathanson, Additive Number Theory. Inverse Problems and the Geometry of Sumsets. Graduate Texts in Mathematics, 165. SpringerVerlag, New York, 1996.

[19] I.Z. Ruzsa, Sums of finite sets, in: Number Theory, Springer-Verlag, New York, 1996, 281-293.

[20] S. Safin, Powers of sets in free groups, http://arxiv.org/abs/1005.1820 (2010)

[21] E. Schenkman, On the norm of a group, Illinois J. Math. 4 (1960), 150152 .

[22] T. Tao and V.Vu, Additive Combinatorics, Cambridge Studies in Advanced Mathematics, 105. Cambridge University Press, Cambridge, 2006.

[23] T. Tao, Product set estimates for non-commutative groups, Combinatorica 28 (2008), 547-594.

[24] R. Winkler, Hartman sets, functions and sequences - a survey. Probability and number theory - Kanazawa 2005, 517-543, Adv. Stud. Pure Math. 49, Math. Soc. Japan, Tokyo, 2007. 